\title{
COMPLÉTUDE ET FLOTS NUL-GÉODÉSIBLES EN GÉOMÉTRIE LORENTZIENNE
}

\author{
PAR PIERRE Mounoud
}

\begin{abstract}
RÉSumÉ. - On étudie la complétude géodésique des flots nul-prégéodésiques sur les variétés lorentziennes compactes, ce qui donne une obstruction à être nul-géodésique. On montre que lorsque l'orthogonal du champ de vecteurs engendrant le flot considéré s'intègre en un feuilletage $\mathcal{F}$, la complétude du flot se lit sur l'holonomie de $\mathcal{F}$. On montre ainsi qu'il n'existe pas de flots nul-géodésiques lisses sur $S^{3}$. On montre aussi qu'un 2-tore lorentzien est nul-complet si et seulement si ses feuilletages de type lumière sont $\mathcal{C}^{0}$ linéarisables.

ABStRaCt (Geodesic completeness of null-pregeodesic flows on compact Lorentz manifold in Lorentzian geometry)

We study geodesic completeness of null-pregeodesic flows on compact Lorentz manifold, obtaining an obstruction to be null-geodesic. We show that when the orthogonal distribution to the vectorfield generating the considered flow integrates into a foliation $\mathcal{F}$, the completeness of the flow can be read on the holonomie of $\mathcal{F}$. We obtain this way that there are no smooth null-geodesic flows on $S^{3}$. We also prove that a Lorentzian 2-torus is null-complete if and only if its lightlike foliations are both $\mathcal{C}^{0}$ linearisable.
\end{abstract}

\section{Introduction}

Ce travail est à l'intersection de deux problèmes : le premier est l'étude des feuilletages géodésibles de dimension 1, le second est la complétude géodésique

Texte reçu le 28 mai 2003, révisé le 15 octobre 2003, accepté le 19 décembre 2003

Pierre Mounoud, Université d'Avignon, Laboratoire d'analyse non linéaire et géométrie, 33 rue Louis Pasteur, 84000 Avignon (France) • E-mail : pierre.mounoud@univ-avignon.fr Classification mathématique par sujets (2000). — 53C50, 53C12, 53C22.

Mots clefs. — Flot nul-géodésible, complétude géodésique. 
des variétés lorentziennes. On supposera ces feuilletages orientés, ce qui est possible quitte à prendre un revêtement à deux feuillets; on les appellera alors flots sans qu'il soit question d'une paramétrisation.

En géométrie riemannienne, un flot $\phi$ est dit géodésible s'il existe une métrique riemannienne $g$ telle que les feuilles de $\phi$ sont des géodésiques géométriques, c'est-à-dire non paramétrées, de $g$ ( $c f$. [11]). Si on paramètre le flot par la longueur d'arc, on voit que $\phi$ possède alors un paramétrage géodésique.

La situation est plus subtile en géométrie pseudo-riemannienne.

- Un flot $\phi$ dont les feuilles sont des géodésiques géométriques d'une métrique $h$ sera dit prégéodésique.

- On dira que $\phi$ est géodésique si de plus $\phi$ possède un paramétrage géodésique.

C'est bien entendu la présence de géodésiques de type lumière qui empêche de paramétrer par la longueur d'arc et qui crée cette difficulté supplémentaire.

On s'est intéréssé dans cet article aux flots nul-géodésibles ${ }^{(1)}$ en géométrie lorentzienne, c'est-à-dire pour lesquels il existe une métrique de signature $(-,+, \ldots,+)$ telle que les feuilles du flot sont toutes des géodésiques de type lumière et qu'il existe un paramétrage géodésique du flot.

Le lien avec la complétude est fait par la remarque suivante : si le flot $\phi$ est (nul)-géodésique, le paramétrage des géodésiques portées par $\phi$ est le même que celui d'un flot défini sur toute la variété; si celle-ci est compacte, alors ces géodésiques sont forcément complètes. La non complétude géodésique d'un flot prégéodésique est donc une obstruction à être géodésique ou inversement sa complétude géodésique en est une version faible ( $c f . \S 5)$.

Ainsi sur une surface lorentzienne les directions de type lumière sont toujours prégéodésiques mais les géodésiques de type lumière ne sont pas toujours complètes (cf. [1]). Par exemple, le tore de Clifton-Pohl, obtenu en quotientant $\left(\mathbb{R}^{2} \backslash\{(0,0)\}, \mathrm{d} x \mathrm{~d} y /\left(x^{2}+y^{2}\right)\right)$ par l'homothétie de rapport 2 , possède bien deux flots nul-prégéodésiques mais aucun flot nul-géodésique car toutes ses géodésiques de type lumière sont incomplètes.

Les flots nul-géodésibles n'ont, à notre connaissance, été étudiés que dans l'article [2], alors que la complétude a sucité de nombreux travaux. Nous ne citerons que [1] et [6] pour la similitude des points de vue. L'article de Y. Carrière et L. Rozoy [1] a fortement inspiré notre approche, c'est de là que vient l'idée ( $c f$. proposition 3.1) que, pour les flots nul-prégéodésiques, la complétude géodésique est une propriété différentielle d'un feuilletage.

Les flots nul-prégéodésiques sont notamment rencontrés comme direction orthogonale des feuilletages de codimension 1 et de type lumière. Le lecteur

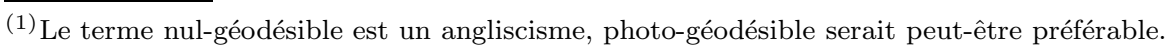
TOME $132-2004-\mathrm{N}^{\mathrm{O}} 3$ 
pourra trouver dans [12] une présentation des situations où l'on rencontre certains de ces feuilletages : ceux dont les feuilles sont totalement géodésiques. Ces seuls exemples suffiraient pour motiver l'étude des flots nul-prégéodésiques.

Les flots et les métriques étudiés dans cet article sont lisses (de classe $\mathcal{C}^{2}$ suffit en fait) ; le cas des flots continus, lui aussi intéressant, nécessiterait une approche différente. Précisons que l'on s'est restreint aux flots nul-prégéodésiques dont la distribution orthogonale est intégrable. Toutefois ceci n'est pas une restriction si la variété est de dimension 2 ou 3 (cf. lemme 2.2). Le fait central de notre travail est la mise en évidence du lien direct entre l'holonomie de ce feuilletage de codimension 1 et la complétude géodésique du flot (lemme 2.4). C'est ce qui nous permet d'obtenir notamment les résultats suivant.

Corollaire 3.4. - Un 2-tore lorentzien est nul-complet (c'est-à-dire toutes ses géodésiques de type lumière sont complètes) si et seulement si ses feuilletages de type lumière sont $\mathcal{C}^{0}$-conjugués à des feuilletages linéaires.

Il s'agit d'une amélioration des résultats de Y. Carrière et L. Rozoy dans [1]. Dans le cas des variétés de dimension 3, une étude des propriétés dynamiques des flots tangents à un feuilletage de Reeb permet de montrer le théorème suivant.

THÉORÈme 4.1. - Soit $M$ une variété lorentzienne de dimension 3 munie d'un flot nul-prégéodésique $\phi$. Si le feuilletage défini par $T \phi^{\perp}$ contient une composante de Reeb, alors $\phi$ n'est pas géodésiquement complet.

On peut appliquer une double lecture à ce théorème : d'une part il fournit une assez importante famille de métriques incomplètes sur la sphère $S^{3}$, en fait sur de nombreuses variétés de dimension 3 (cf. [6] pour une autre famille), d'autre part il clôt la question de l'existence de flots nul-géodésibles sur la sphère (car ils doivent être géodésiquement complets).

L'auteur souhaite remercier le rapporteur pour sa lecture attentive et ses suggestions.

\section{Définitions et propriétés locales.}

On se placera dans toute la suite sur une variété compacte $M$, les métriques seront lisses et les feuilletages seront tous de classe $\mathcal{C}^{2}$. Commençons par préciser les termes que nous allons employer. Tout d'abord, notons que le terme flot désigne un feuilletage de dimension 1 orienté sans aucune idée de paramétrisation. Ensuite rappelons que sur une variété lorentzienne $(M, h)$ un vecteur tangent $v$ est dit de type lumière si $h(v, v)=0$; par extension on parlera aussi de géodésiques de type lumière et on dira qu'une sous-variété est de type lumière si la restriction de $h$ à son tangent est partout dégénérée. Enfin on notera $D$ la connexion de Levi-Civita de $h$. 
DÉfinition 2.1. — Soit $(M, h)$ une variété lorentzienne.

- Un flot $\phi$ sera dit nul-prégéodésique si ses feuilles sont des géodésiques non paramétrées de type lumière de $h$. Le flot $\phi$ sera dit nul-géodésique si, de plus, il est possible de le paramétrer globalement de façon géodésique.

- Un flot prégéodésique sera dit géodésiquement complet si toutes les géodésiques contenues dans ses feuilles sont complètes (c'est-à-dire définies pour tout temps).

Un flot $\phi$ sur $M$ sera dit nul-(pré)géodésible s'il existe une métrique sur $M$ pour laquelle il est nul-(pré)géodésique.

Avant tout donnons le critère suivant qui assure qu'un flot est nul-prégéodésible (on trouve un critère analogue pour le cas riemannien dans [11]).

LEMME 2.2. - Un flot $\phi$ sur $M$ est nul-prégéodésible si et seulement si il existe une distribution $\mathcal{H}$ d'hyperplans transversalement orientable tangente $\grave{a}$ $\phi$ et préservée par $\phi$.

Démonstration. — L'orientabilité de $\phi$ nous permet de considérer une trivialisation $X$ du tangent à $\phi$. Rappelons d'une part qu'une distribution $\mathcal{H}$ est préservée par $\phi$ si et seulement si le crochet d'un champ de vecteurs inclus dans $\mathcal{H}$ avec $X$ est encore dans $\mathcal{H}$. D'autre part que le flot $\phi$ est prégéodésique pour une métrique $h$ de connexion de Levi-Civita $D$ si et seulement si $D_{X} X$ est colinéaire à $X$. Un calcul direct suffit dès lors pour montrer le lemme.

En complément donnons le critère suivant, que l'on trouve dans l'article [2] de C. Chicone et P. Ehrlich, qui ressemble beaucoup au lemme 2.2 mais qui concerne cette fois les flots nul-géodésibles.

Proposition 2.3 (cf. [2]). — Un flot $\phi$ sur un variété $M$ est nul-géodésible si et seulement si il existe un champ de vecteurs $X$ partout non nul et tangent $\grave{a}$ $\phi$ et une 1-forme $\omega$ partout non nulle tels que $i_{X} \omega=0$ et $i_{X} \mathrm{~d} \omega=0$.

Démonstration. - On vérifie par un calcul direct qu'un champ de vecteurs de type lumière $X$ vérifie $D_{X} X=0$ si et seulement si la 1 -forme $\omega=h(X,$. vérifie les hypothèses de l'énoncé.

Parmis les flots vérifiant les hypothèses du lemme 2.2 , il y a notamment les flots tangents à un feuilletage de codimension 1. Dans la suite, on se restreindra à ce cas ; remarquons que cette condition sera toujours vérifiée si la variété est de dimension 2 ou 3. Sous cette hypothèse on a une bonne description locale de la situation. Localement, dans une carte adaptée aux feuilletages, la métrique vérifie les hypothèses du lemme suivant.

LEMME 2.4. - Soit $h$ une métrique lorentzienne sur un ouvert $U$ de $\mathbb{R}^{m+1}$ telle que $\partial_{x_{0}}$ soit nul-prégéodésique, $\partial_{x_{1}}, \ldots, \partial_{x_{m-1}}$ soient orthogonaux à $\partial_{x_{0}}$. On pose $\alpha=h\left(\partial_{x_{0}}, \partial_{x_{m}}\right)$. Soit $t$ le temps où la géodésique partant du point 
$(0, \ldots, 0)$ au temps $t_{0}$ et à la vitesse $\left(\dot{x}\left(t_{0}\right), 0, \ldots, 0\right)$ atteint le point $(a, 0, \ldots, 0)$. On $a$

$$
t-t_{0}=\frac{1}{\alpha(0, \ldots, 0) \dot{x}\left(t_{0}\right)} \int_{0}^{a} \alpha(x, 0 \ldots, 0) \mathrm{d} x .
$$

Démonstration. — On rappelle les équations d'Euler-Lagrange (cf. [4]) ; on désigne par $\dot{x}(t)$ la dérivée de $x(t)$ par rapport à $t$ :

$$
\ddot{x}_{i}(t)+\sum_{j, k} \Gamma_{j, k}^{i}(\gamma(t)) \dot{x}_{k}(t) \dot{x}_{j}(t)=0 \quad(0 \leq i \leq m),
$$

où $\Gamma_{j, k}^{i}$ désigne les symboles de Christoffel de $h$. La direction $\partial_{x_{0}}$ étant prégéodésique, l'équation se simplifie. En effet, on a $\dot{x}_{i}(t)=0$ pour $1 \leq i \leq m$; après calcul de $\Gamma_{1,1}^{1}$, on obtient l'équation

$$
\alpha \ddot{x}_{0}(t)+\left(\partial_{x_{0}} \alpha\right)\left(\dot{x}_{0}(t)\right)^{2}=0
$$

où $\alpha=h\left(\partial_{x_{0}}, \partial_{x_{m}}\right)$. On remarque que l'on obtient la même équation que Carrière et Rozoy dans [1]. L'équation se ramène à $\alpha(x(t)) \dot{x}_{0}(t)=1 / C$, où $C$ est une constante. On peut donc récupérer le paramètre affine $t$ de la géodésique en écrivant $\mathrm{d} t=\mathrm{C} \alpha \mathrm{d} x_{0}$ et en intégrant. On obtient l'égalité désirée : en effet $C$ est donnée par la vitesse initiale de la géodésique, on a $C^{-1}=\alpha(0, \ldots, 0) \dot{x}_{0}\left(t_{0}\right)$.

\section{Complétude et pseudo-groupe d'holonomie}

On se restreint dorénavant au cas particulier où la distribution orthogonale du flot nul-prégéodésique est intégrable. On montre alors :

Proposition 3.1. - Soient $\mathcal{F}$ un feuilletage transversalement orientable de codimension 1 et $\phi$ un flot inclus dans $\mathcal{F}$. Soit $h$ une métrique lorentzienne telle que $\phi$ est de type lumière et que $(T \phi)^{\perp}=T \mathcal{F}$. Alors le flot $\phi$ est nulprégéodésique et la complétude géodésique de $\phi$ ne dépend pas du choix de $h$.

Cette proposition montre que, dans ce cas du moins, la complétude géodésique d'un flot nul-prégéodésique est une propriété des feuilletages.

Démonstration. - On considère à nouveau une trivialisation $X$ de $T \phi$. On se donne maintenant un atlas fini $\left\{U^{i}, 1 \leq i \leq p\right\}$ chaque ouvert étant sur le modèle du lemme 2.4 : chaque $U^{i}$ est muni des coordonnées $\left(x^{i}\right)$ telles que $\partial_{x_{0}^{i}}=X$ et $\partial_{x_{\ell}^{i}}$ est orthogonal à $X$ si $1 \leq \ell \leq m-1$. On désigne par $\Psi^{i j}=$ $\left(\Psi_{0}^{i j}, \ldots, \Psi_{m}^{i j}\right)$ le changement de cartes de $U^{i}$ vers $U^{j}$ (si l'intersection est non vide) envoyant les coordonnées $\left(x^{i}\right)$ sur les coordonnées $\left(x^{j}\right)$. Sur chaque $U^{i}$, on pose $\alpha^{i}=h\left(\partial_{x_{0}^{i}}, \partial_{x_{m}^{i}}\right)$. On a $\alpha^{j}=\left(\partial_{x_{m}^{i}} \Psi_{m}^{i j}\right) \alpha^{i}$.

On découpe maintenant la géodésique $\gamma$ en les points $a_{n}$ de telle sorte qu'entre $a_{n}$ et $a_{n+1}$ la courbe reste dans l'ouvert $U^{i_{n}}, i_{n} \in\{1, \ldots, p\}$ (ainsi 
$\left.a_{n+1} \in U^{i_{n}} \cap U^{i_{n+1}}\right)$. On a donc une suite $\left\{i_{1}, \ldots, i_{n}, \ldots,\right\}$ donnant la liste des cartes successivement traversées par $\gamma$ lorsque $t$ augmente. Sur un ouvert $U^{i_{k}}$, on a $\mathrm{d} t=C^{i_{k}} \alpha^{i_{k}} \mathrm{~d} x_{0}^{i_{k}}$. En intégrant on obtiendrait la variation de $t$ entre $a_{k}$ et $a_{k+1}$ à condition de se donner $C^{i_{k}}$. On va voir comment la donnée de $\dot{\gamma}(0)$ impose la valeur de $C^{i_{k}}$.

Pour tout entier $k$, on note $t_{k}$ le réel vérifiant $\gamma\left(t_{k}\right)=a_{k}$. Le point $a_{k}$ étant à l'intersection de $U^{i_{k}}$ et de $U^{i_{k+1}}$, on peut relier $C^{i_{k}}$ et $C^{i_{k+1}}$ grâce à ce point. On a

$$
\left(C^{i_{k}}\right)^{-1}=\alpha^{i_{k}}\left(a_{k}\right) \dot{x}_{0}^{i_{k}}\left(t_{k}\right), \quad\left(C^{i_{k+1}}\right)^{-1}=\alpha^{i_{k+1}}\left(a_{k}\right) \dot{x}_{0}^{i_{k+1}}\left(t_{k}\right) .
$$

Notre choix d'atlas entraîne que $\dot{x}_{0}^{i_{k+1}}\left(t_{k}\right)=\dot{x}_{0}^{i_{k}}\left(t_{k}\right)$. On a donc :

$$
\begin{gathered}
\frac{C^{i_{k+1}}}{C^{i_{k}}}=\frac{\alpha^{i_{k}}\left(a_{k}\right)}{\alpha^{i_{k+1}}\left(a_{k}\right)} . \\
\text { Or, } \alpha^{i_{k}}\left(a_{k}\right)=\left(\partial_{x_{m}^{i_{k+1}}} \Psi_{m}^{i_{k+1} i_{k}}\right)\left(a_{k}\right) \alpha^{i_{k+1}}\left(a_{k}\right) . \text { On a donc } \\
C^{i_{k+1}}=C^{i_{k}}\left(\partial_{x_{m}^{i_{k}}} \Psi_{m}^{i_{k} i_{k+1}}\right)\left(a_{k}\right) .
\end{gathered}
$$

En répétant ainsi de suite on a alors

$$
C^{i_{k+1}}=C^{i_{0}}\left(\partial_{x_{m}^{i_{1}}} \Psi_{m}^{i_{2} i_{1}}\right)\left(a_{1}\right) \cdots\left(\partial_{x_{m}^{i_{k}}} \Psi_{m}^{i_{k+1} i_{k}}\right)\left(a_{k}\right) .
$$

On est maintenant capable de sommer les variations de $t$ sur chaque segment de géodésique $\left[a_{k}, a_{k+1}\right]$. La géodésique est complète si et seulement si $t$ tend vers l'infini lorsque l'on parcourt $\gamma$, c'est-à-dire si la somme des variations de $t$ est infinie, c'est-à-dire si

(*) $\quad \sum_{k=1}^{\infty}\left(\partial_{x_{m}^{i_{1}}} \Psi_{m}^{i_{2} i_{1}}\right)\left(a_{1}\right) \cdots\left(\partial_{x_{m}^{i_{k}}} \Psi_{m}^{i_{k+1} i_{k}}\right)\left(a_{k}\right) \int_{a_{k}}^{a_{k+1}} \alpha^{i_{k}}\left(x, y_{k}, z_{k}\right) \mathrm{d} x=+\infty$,

et si on a la même relation lorsque l'on parcourt $\gamma$ dans l'autre sens. On peut choisir l'atlas et les points $a_{k}$ de telle sorte que les différences $a_{k+1}-a_{k}$ soient bornées. Les fonctions $\alpha^{i}$ étant également bornées loin de 0 la convergence de cette somme ne dépend pas des valeurs des intégrales et donc pas du choix de la métrique. Seuls les choix de $\phi$ et de $\mathcal{F}$ importent.

Il n'est pas surprenant que les $\Psi_{m}^{i_{k} i_{k+1}}$ interviennent dans l'écriture de (*), en effet il s'agit d'éléments du pseudo-groupe d'holonomie de $\mathcal{F}$. Pour une feuille fermée de $\phi$, la formule $\left(^{*}\right)$ dit que la complétude de cette géodésique dépend uniquement de l'holonomie linéaire de la feuille de $\mathcal{F}$ portée par la feuille.

Corollaire 3.2. - On garde les notations de la proposition 3.1. S'il existe $x \in M$ tel que la feuille de $\phi$ passant par $x$, notée $\phi_{x}$, est fermée et si l'holonomie linéaire portée par le lacet $\phi_{x}$ est différente de \pm 1 alors la géodésique dont le lieu coincide avec $\phi_{x}$ est incomplète.

TOME $132-2004-\mathrm{N}^{\mathrm{O}} 3$ 
Démonstration. - On garde les notations de la preuve de la proposition 3.1. La feuille $\phi_{x}$ étant fermée, on peut la recouvrir par deux ouverts sur le modèle du lemme 2.4. On a alors un seul changement de carte $\Psi$ et la dérivée $\left(\partial_{x_{m}} \Psi_{m}\right)(x)$ représente exactement l'holonomie linéaire portée par $\phi_{x}$. La formule $\left(^{*}\right)$ est vérifiée si et seulement si la valeur absolue de cette dérivée est supérieure ou égale à 1 . D'autre part, la formule obtenue en parcourant le lacet dans l'autre sens est vérifiée si $\left|\left(\partial_{x_{m}} \Psi_{m}\right)(x)\right| \leq 1$.

Par contre, il semble difficile de lire la complétude géodésique des courbes non fermées sur l'holonomie de la feuille de $\mathcal{F}$ qui la contient, on se reportera au paragraphe 4.2 pour des exemples. Lorsque $\mathcal{F}$ est donné par une forme fermée le pseudo-groupe d'holonomie est particulièrement simple, il s'agit, à conjugaison près, de translations et on a sans surprise :

Corollaire 3.3. - Si $\mathcal{F}$ est donné par une 1-forme fermée $\omega$ alors $\phi$ est toujours geodésiquement complet

Démonstration. - On note $X$ un champ de vecteurs partout non nul et tangent à $\phi$. Dans de telle condition on trouve facilement une métrique $h$ telle que $D_{X} X=0$ et $h(X, X)=0$, il suffit de prendre $h$ telle que $h(X,)=.\omega$ (voir proposition 2.3). Dès lors il existe une métrique telle que $\phi$ est géodésiquement complet et donc $\phi$ est toujours géodésiquement complet.

La question qui vient immédiatement est : qu'en est-il si on impose seulement à $\mathcal{F}$ d'être sans holonomie? Nous n'avons pour l'instant qu'un résultat en dimension 2 où être sans holonomie revient à être $\mathcal{C}^{0}$-conjugué à un feuilletage linéaire (c'est-à-dire obtenu par suspension d'une rotation). Il s'agit d'une amélioration des résultats de Carrière et Rozoy dans [1].

Corollaire 3.4. - Un 2-tore lorentzien est nul-complet (c'est-à-dire toutes ses géodésiques de type lumière sont complètes) si et seulement si ses feuilletages de type lumière sont $\mathcal{C}^{0}$-conjugués à des feuilletages linéaires.

Démonstration. - Soit $\mathcal{F}$ un feuilletage $\mathcal{C}^{0}$ conjugué à un feuilletage linéaire. Il est obtenu par suspension d'un diffeomorphisme du cercle, il existe donc un feuilletage transverse dont toutes les feuilles sont fermées. Dans l'article de Carrière et Rozoy [1], il est montré que si l'un des feuilletages isotropes est $\mathcal{C}^{0}$-linéaire et l'autre est à feuilles fermées alors la métrique est nul-complète. Ainsi il existe une métrique telle que $\mathcal{F}$ est de type lumière et géodésiquement complet. D'après la proposition 3.1 c'est donc le cas pour toutes les métriques. 


\section{La dimension 3}

Comme on l'a déjà fait remarquer, l'orthogonal d'un flot nul-prégéodésique est toujours intégrable en dimension 3 , on peut donc étudier tous les flots nul-prégéodésiques grâce à la description locale donnée par le lemme 2.4 et la proposition 3.1 est toujours valable.

4.1. La sphère.- Une question naturelle est de savoir ce qu'il se passe lorsque le feuilletage orthogonal contient une composante de Reeb. On montre :

THÉORÈme 4.1. - Soit $M$ une variété lorentzienne de dimension 3 muni d'un flot nul-prégéodésique $\phi$. Si le feuilletage défini par $T \phi^{\perp}$ contient une composante de Reeb alors $\phi$ n'est pas géodésiquement complet.

Démonstration. - Les propriétés des champs de vecteurs tangents aux feuilletages de Reeb ont été étudiées par A. Davis et J.F. Wilson Jr. dans [3]. En s'appuyant sur les travaux de Reinhardt sur les feuilletages du tore [10], ils donnent des propriétés homotopiques du flot restreint à la feuille torique. Ils établissent en particulier l'existence de composantes de Reeb et donc d'orbites fermées sur la feuille torique.

Restreignons-nous donc à une composante de Reeb et regardons les propriétés de la restriction de $\phi$ à $T$ la feuille torique de cette composante. Rappelons qu'un feuilletage sur un tore a un nombre fini de composantes de Reeb (de dimension 1 cette fois) mais éventuellement une infinité de feuilles fermées.

Soient $\tilde{\ell}$ et $\widetilde{m}$ une base de $H_{1}(T)$ telle que $-\tilde{\ell}$ soient la direction asymptotique du feuilletage de Reeb, c'est-à-dire que toute feuille de $\mathcal{F}$ spirale vers $T$ dans la direction $-\tilde{\ell}$. Il existe deux entiers $a$ et $b$ tels que les feuilles fermées de $\phi_{\mid T}$ sont homologues à $-a \tilde{\ell}-b \tilde{m}$ ou à $a \tilde{\ell}+b \tilde{m}$.

On désigne par $p$ (resp. $q$ ) le nombre de composantes de Reeb dont l' $\alpha$-limite (qui est aussi égale à l'w-limite) est homologue à $-a \tilde{\ell}-b \tilde{m}$ (resp. à $a \tilde{\ell}+b \tilde{m})$.

A. Davis et J.F. Wilson ont montré que

$$
p-q=2 \quad \text { et } \quad a=1 .
$$

En particulier la restriction de $\phi$ à cette feuille torique possède au moins deux composantes de Reeb et au moins deux feuilles fermées. (On retrouve le résultat de A. Zeghib ( $c f$. [12]) affirmant qu'un feuilletage de type lumière de codimension 1 et totalement géodésique en dimension 3 ne peut pas contenir de composante de Reeb. En effet, la restriction de son orthogonal à une feuille est riemannien et donc linéaire.)

On peut cependant en déduire quelque chose de plus intéressant pour nous : il existe deux composantes de Reeb de même direction qui sont consécutives, c'est-à-dire séparées uniquement par des composantes obtenues par suspension et dont l' $\alpha$-limite est homotope à $-\tilde{c} f-b \tilde{m}$. Notons que pour une composante obtenue par suspension l' $\alpha$-limite est égale à l'opposé de l'w-limite.

TOME $132-2004-\mathrm{N}^{\mathrm{O}} 3$ 
Si ces deux composantes sont vraiment consécutives, si la composante voisine est « dans le bon sens » ou si deux composantes obtenues par suspension voisines sont de sens opposés on a les situations suivantes, où le lecteur recon-
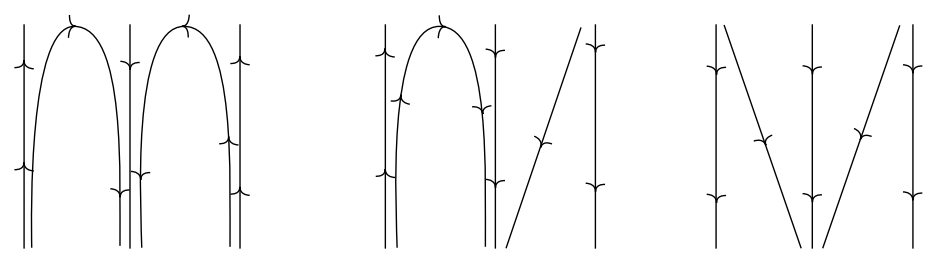

naitra les composantes de Reeb, représentées par les arches, les composantes obtenues par suspension, représentées par les flèches diagonales et les feuilles fermées représentées par les flèches verticales. Le flot $\phi$ a donc une orbite fermée attractive. Nous avons, au passage, choisi une orientation pour $\phi$.

Mais on peut aussi avoir une infinité de courbes fermées entre les deux composantes de Reeb. On peut avoir la situation suivante c'est-à-dire une accumu-

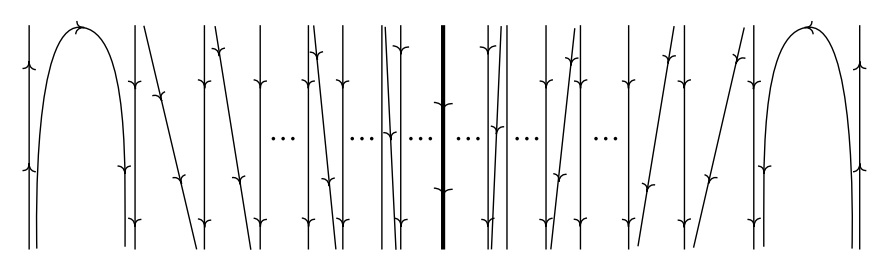

lation de composantes obtenues par suspension entre les deux composantes de Reeb, les composantes obtenues par suspension pouvant être à feuilles fermées. Il n'y a pas d'orbite fermées attractive au sens classique; cependant l'orbite fermée centrale représentée en gras a une propriété remarquable : les orbites non fermées voisines seront encore toutes attirée, en un sens faible, vers cette orbite (elles ne viendront pas s'accumuler sur cette orbite). Même dans ce cas, la situation va nous permettre de construire un voisinage, dans $M$, de l'orbite remarquable obtenue, invariant par $\phi$ dans une direction. On nomme $\gamma$ cette orbite.

Replaçons-nous dans $M$. Prenons un demi-voisinage $U$ de $\gamma$ délimité par la feuille $T$ et intérieur à la composante de Reeb. Soit $S$ une surface dans $U$ transverse à $\phi$ (et donc à $\mathcal{F}$ ) qui coupe $\gamma$. On considère $\mathcal{T}$ l'application de premier retour associée à $\phi$ (rappelons que $\phi$ est orienté). La trace de la feuille torique sur $S$ est une courbe, l'orbite fermée donne un point fixe $u$. D'après ce qui précède il y a de part et d'autre de $u$ des points $w$ tels que $\mathcal{T}(w)$ est plus proche de $u$ que $w$. De plus les feuilles planes de $\mathcal{F}$ spiralent vers $T$ dans la direction $-\tilde{\ell}$. Considérons des coordonnées feuilletées qui envoient $u$ sur $(0,0,0)$ 
et telles que les points de $T$ ont des coordonnées de la forme $(x, y, 0)$. Soit $w=\left(w_{1}, w_{2}, w_{3}\right)$ un point de $S$ et $\mathcal{T}(w)=\left(\mathcal{T}_{1}(w), \mathcal{T}_{2}(w), \mathcal{T}_{3}(w)\right)$ son image par $\mathcal{T}$. Bien sûr si $w_{3}=0$ on a $\mathcal{T}_{3}(w)=0$. Comme $a=1$, on a $\mathcal{T}_{3}(w)<w_{3}$ car les feuilles spiralent justement dans la direction $-\tilde{\ell}$. L'application $\mathcal{T}$ étant continue on en déduit l'existence de $S^{\prime} \subset S$ invariante par $\mathcal{T}$ et donc d'un voisinage $U^{\prime} \subset U$ invariant par $\phi$ dans le sens positif.

Grâce à ce travail et au lemme 2.4 on rejoint la démonstration de [1]. On peut voir $U^{\prime}$ muni de la restriction de la métrique $h$ comme le quotient de $\left\{(x, y, z) \in \mathbb{R}^{3} \mid x>0,0 \leq y \leq y_{0}, 0 \leq z \leq z_{0}\right\}$, avec $\partial_{x}$ colinéaire à $T \phi$ et $\partial_{y}$ orthogonal à $T \phi$, par l'action définie par $\Psi(x, y, z)=\left(x+1, \Psi_{2}(x, y, z), \Psi_{3}(z)\right)$ avec $\Psi_{3}(0)=0$ et $\Psi_{3}(z)<z$ sinon. On pose $\alpha=h\left(\partial_{x}, \partial_{z}\right)$. On a $\alpha(\Psi(x, y, z))=$ $\partial_{z} \Psi_{3}(z) \alpha(x, y, z)$ car la métrique relevé est invariante par $\phi$. La géodésique est incomplète si elle est parcourue en un temps fini. Grâce au lemme 2.4 on peut calculer la variation de $t$. La géodésique partant du point $(x, y, z)$ est incomplète si et seulement si la série suivante converge :

$$
\sum_{k} \partial_{z}\left(\Psi_{3}^{k}(z)\right) \int_{0}^{1} \alpha\left(x, \Psi_{2}^{k}(x, y, z), \Psi_{3}^{k}(z)\right) \mathrm{d} x<+\infty .
$$

Comme la fonction $\alpha$ est bornée loin de 0 cette série converge si et seulement si la série

$$
\zeta(z)=\sum_{k} \partial_{z}\left(\Psi_{3}^{k}(z)\right)
$$

converge.

On reprend l'argument final de Carrière et Rozoy : on remarque que les itérées de $J=] \Psi_{3}(z), z\left[\right.$ sont disjoints $(z \neq 0)$ et que donc la série $\sum_{k} \lambda\left(\Psi_{3}^{k}(J)\right)$ est convergente ( $\lambda$ est la mesure de Lebesgue). Comme elle s'obtient en intégrant $\zeta$ sur $J$ la série converge pour presque tout $z \in J$. On obtient donc des géodésiques incomplètes.

Corollaire 4.2. - Il n'existe pas sur $S^{3}$ ou $S^{2} \times S^{1}$ (plus généralement sur toute variété compacte de dimension 3 dont le revêtement universel n'est pas $\mathbb{R}^{3}$ ) de flots nul-géodésibles.

Démonstration. - D'une part, comme on l'a décrit en introduction, un flot nul-géodésique est géodésiquement complet et d'autre part, d'après les travaux de Novikov et Palmera ( $c f$. [8] et [9]), les feuilletages de codimension 1 sur ces variétés contiennent forcément des composantes de Reeb. D'après le théorème 4.1 ces deux situations ne peuvent coexister.

Comme tout champ de plans sur $S^{3}$ est parallélisable, tout feuilletage de codimension 1 de $S^{3}$ admet un sous-feuilletage. Il est assez facile de fabriquer une métrique qui rende le flot nul-prégéodésique, il y en a même beaucoup. Ainsi à tout feuilletage on peut associer des métriques ayant un flot nul-prégéodésique

TOME $132-2004-\mathrm{N}^{\mathrm{O}} 3$ 
et donc incomplètes. Ceci donne une famille assez vaste de métriques incomplètes sur la sphère. On peut faire la même construction à partir de n'importe quel feuilletage contenant une composante de Reeb à condition de vérifier qu'il existe bien un sous-feuilletage.

4.2. Autres exemples intéressants. - Pour faciliter l'exposition nous commençons par donner le lemme suivant :

Lemme 4.3. - Soit $\mathcal{F}_{1}$ et $\mathcal{F}_{2}$ deux feuilletages de codimension 1 partout transverses et soit $\phi$ le flot défini par l'intersection. Si de plus $\phi$ possède des trajectoires fermées attractives sur $\mathcal{F}_{1}$, alors pour toute métrique $h$ telle que $T \phi^{\perp}=T \mathcal{F}_{2}$, le flot $\phi$ est géodésiquement incomplet.

Démonstration. - On a donc une courbe intégrale de $\phi$ qui s'accumule sur une feuille qui n'est pas dans $\phi^{\perp}$. C'est exactement ce qui fait fonctionner la preuve du théorème 4.1. Il ne reste plus qu'à reprendre le dernier paragraphe de cette preuve.

1) Un feuilletage de $M=\mathbb{T}^{2} \times[0,1]$ de codimension 1 tangent au bord et sans autre feuille compacte est $\mathcal{C}^{0}$-conjugué à un feuilletage défini par une action localement libre de $\mathbb{R}^{2}(c f$. [7]). Nous allons regarder ce que l'on peut dire dans le cas différentiable. On considère le feuilletage défini par la 1-forme $\Omega=\mathrm{d} r+\varphi(r) \omega$ où $\omega$ est une forme linéaire sur le tore et $\varphi$ une fonction ne s'annulant qu'en 0 et 1 . Soit $X$ un champ de vecteur linéaire du tore vérifiant $i_{X} \omega=0$. On note $\widetilde{X}$ sont prolongement trivial à $M$. On a $i_{\widetilde{X}} \Omega=0$ et comme $\omega$ est fermée on a $i_{\widetilde{X}} \mathrm{~d} \Omega=0$. D'après la proposition 2.3, il existe une métrique telle que $\widetilde{X}$ soit de type lumière et que $D_{\widetilde{X}} \widetilde{X}=0$. Ainsi si ce champ est pris comme orthogonal au feuilletage, le flot associé sera géodésiquement complet.

Prenons $Y$ sur le tore dont toutes les courbes sont fermées et qui vérifie $i_{Y} \omega=1$. Cette fois prenons $\widetilde{Y}=-\varphi(r) \partial_{r}+Y$ ce champ annule bien $\Omega$. Il est facile de vérifier que $\widetilde{Y}$ et $\partial_{r}$ sont en involution et définissent un feuilletage $\mathcal{G}$. Il est clair que les trajectoires de $\widetilde{Y}$ s'accumulent sur les trajectoires du bord. On peut appliquer le lemme 4.3. Si on choisit $\Omega$ de telle sorte que $\mathcal{F}$ ait une holonomie linéaire non triviale alors on peut aussi conclure par le corollaire 3.2. Ainsi si $\widetilde{Y}$ est choisi comme direction de type lumière, le flot associé est géodésiquement incomplet.

Que l'on choisisse $\widetilde{X}$ ou $\tilde{Y}$ comme direction de lumière, si $\tilde{X} \cdot h(\tilde{Y}, \tilde{Y})=0$ et si $\tilde{Y} \cdot h(\widetilde{X}, \widetilde{X})=0$, il est facile de vérifier que la condition $[\tilde{X}, \tilde{Y}]=0$ entraine que les feuilles de $\mathcal{F}$ sont totalement géodésiques : ce n'est donc pas une condition suffisante pour assurer la complétude. 
2) Les flots d'Anosov dont les feuilletages stables et instables faibles sont lisses sont une famille classique de flots tangents à un feuilletage de codimension 1. On peut se demander s'ils peuvent être rendus nul-prégéodésiques et géodésiquement complets. Ces feuilletages stables et instables sont les seuls auquels l'Anosov soit tangent. Les feuilles de ces feuilletages sont des cylindres ou des plans. On sait d'autre part que toutes les feuilles cylindriques ont une holonomie linéaire non triviale. Un flot d'Anosov possède des orbites fermées, forcément à l'intersection de deux cylindres. Ces lacets portent obligatoirement l'holonomie de la feuille et d'après le corollaire 3.2 ces lacets géodésiques sont tous non complets. Il n'existe donc pas de métriques qui rende un flot d'Anosov nul-prégéodésique et géodésiquement complet.

Ainsi, le flot géodésique d'une surface hyperbolique n'est pas nul-géodésible, mais son flot horocyclique, qui est aussi son feuilletage fortement stable, l'est (il est nul-géodésique pour la métrique induite par la forme de Killing). Dans le cas des flots d'Anosov obtenus par suspension le feuilletage fortement stable est encore nul-geodesible (pour la métrique plate invariante par le flot d'Anosov). Il est intéressant de noter que ce feuilletage est obtenu par intersection transverse de deux feuilletages de codimension 1 : le feuilletage faiblement stable de l'Anosov et le feuilletage en tore de $T_{A}^{3}$. Ce n'est donc pas une propriété incompatible avec être nul-géodésible ( $c f$. lemme 4.3).

D'après l'article d'Etienne Ghys [5], les feuilletages de codimension 1 sans composantes de Reeb du tangent unitaire à une surface hyperbolique sont tous isotopes. Sur cette variété, la question de connaitre tous les flots nul-géodésibles est alors plus raisonnable. Le corollaire 3.2 entraine qu'un tel flot ne peut avoir de feuilles fermées. On peut se demander si les flots horocycliques sont les seuls exemples.

\section{Discussion sur les flots nul-géodésibles}

Les flots géodésiquement complets que l'on a rencontrés possédaient pour la plupart une paramétrisation géodésique. On peut donc se demander si tout flot nul-prégéodésique et géodésiquement complet est nul-géodésible. Soit $Y$ une trivialisation du tangent au flot; rappelons la proposition 2.3 : il existe une métrique $h$ telle que $h(Y, Y)=0$ et que $D_{Y} Y=0$ si et seulement si il existe une 1-forme $\omega$ telle que $i_{Y} \omega=0$ et que $i_{Y} \mathrm{~d} \omega=0$. En dimension 2, $\omega$ est alors tout simplement fermée et donc le feuilletage défini par $Y$ est $\mathcal{C}^{\infty}$ conjugué à un feuilletage linéaire. Si l'un des feuilletages de type lumière, $\mathcal{F}$, de $h$ est $\mathcal{C}^{\infty}$-conjugué à un feuilletage linéaire il est donné par une 1-forme fermée $\omega$ et $Y=\omega^{\sharp}$ vérifie $D_{Y} Y=0$. Ainsi toute métrique conformément plate possède donc deux champs de vecteurs de type lumière géodésiques. Cependant si $\mathcal{F}$ n'est que $\mathcal{C}^{1}$-linéarisable, $\omega$ existe toujours mais n'est que $\mathcal{C}^{1}$; cependant $Y=\omega^{\sharp}$ vérifie toujours $D_{Y} Y=0$ mais $Y$ n'est que de classe $\mathcal{C}^{1}$ (pour le voir on

TOME $132-2004-\mathrm{N}^{\mathrm{O}} 3$ 
se ramène au cas précédent par un difféomorphisme $\mathcal{C}^{1}$ ). Ceci redémontre le fait qu'un tore $\mathcal{C}^{1}$ conformément plat est nul complet. On a vu qu'il y a toujours complétude dans le cas $\mathcal{C}^{0}$ mais existe-t-il alors un champ de vecteurs $Y$ de classe $\mathcal{C}^{0}$ tel que $D_{Y} Y=0$ ? On peut demander plus généralement :

Question. - Soit $\phi$ un flot nul-prégéodésique géodésiquement complet; $\phi$ estil $\mathcal{C}^{0}$-nul-géodésible?

\section{BIBLIOGRAPHIE}

[1] Carrière (Y.) \& Rozoy (L.) - Complétude des métriques lorentziennes de $T^{2}$ et difféomorphismes du cercle, Bol. Soc. Brasil. Mat. (N.S.), t. 25 (1994), no. 2, pp. 223-235.

[2] Chicone (C.) \& Ehrlich (P.) - Lorentzian geodesibility, in Differential topology - geometry and related fields, and their applications to the physical sciences and engineering, Teubner-Texte Math, vol. 76, Teubner, 1985, pp. 5-99.

[3] Davis (A.) \& Wilson (F.W.) - Vector fields tangent to foliations. I : Reeb foliations, J. Differ. Equations, t. 11 (1972), pp. 491-498.

[4] Gallot (S.), Hullin (D.) \& Lafontaine (J.) - Riemannian geometry, $2^{\mathrm{e}}$ éd., Universitext, Springer-Verlag, 1990.

[5] Ghys (E.) - Rigidité différentiable des groupes fuchsiens, Inst. Hautes Études Sci. Publ. Math., t. 78 (1994), pp. 163-185.

[6] Guediri (M.) \& Lafontaine (J.) - Sur la complétude des variétés pseudo-riemanniennes, J. Geom. Phys., t. 15 (1995), no. 2, pp. 150-158.

[7] Moussu (R.) \& Roussarie (R.) - Relations de conjugaison et de cobordisme entre certains feuilletages, Inst. Hautes Études Sci. Publ. Math., t. 43 (1974), pp. 142-168.

[8] Novikov (S.P.) - The topology of foliations, Trudy Moskov. Mat. Ob., t. 14 (1965), pp. 248-278, AMS Transl. (1967), pp. 286-304.

[9] Palmera (F.) - Open manifolds foliated by planes, Annals of Math., t. 107 (1978), pp. 109-131.

[10] Reinhart (B.L.) - Line element on the torus, Amer. J. Math., t. 81 (1959), pp. 617-631.

[11] Sullivan (D.) - A foliation of geodesics is characterized by having no 'tangent homologies', J. Pure Appl. Algebra, t. 13 (1978), no. 1, pp. 101104.

[12] Zeghib (A.) - Geodesic foliations in Lorentz 3-manifolds, Comment. Math. Helv., t. 74 (1999), no. 1, pp. 1-21. 